\title{
O ADVENTO DAS INCUBADORAS E OS CUIDADOS DE ENFERMAGEM AOS PREMATUROS NA PRIMEIRA METADE DO SÉCULO XX ${ }^{1}$

\author{
THE ADVENT OF INCUBATORS AND NURSING CARE TO PREMATURE BABIES IN THE FIRST \\ HALF OF THE TWENTIETH CENTURY
}

\section{LA LLEGADA DE LAS INCUBADORAS Y LOS CUIDADOS DE ENFERMERÍA A LOS PREMATUROS EN LA PRIMERA MITAD DEL SIGLO XX}

\author{
Isabel Cristina dos Santos Oliveira
}

\begin{abstract}
${ }^{1}$ Este estudo faz parte do relatório de pesquisa elaborado pela bolsista de iniciação científica/CNPq Aline da Costa Barbosa e está inserido no Projeto Integrado de Pesquisa/CNPq "A prática da enfermagem nos hospitais pediátricos: a influência da tecnologia nos anos 70", coordenado pela professora doutora Isabel Cristina dos Santos Oliveira.

${ }^{2}$ Doutora em Enfermagem. Professor a Adjunto IV do Departamento de Enfermagem Médico-Cirúrgica da Escola de Enfermagem Anna Nery da Universidade Federal do Rio de Janeiro. Pesquisadora/CNPq.
\end{abstract}

PALAVRAS-CHAVE:

Enfermagem neonatal. Incubadoras-história.

Prematuro.

\section{KEYWORDS:}

Neonatal nursing. Incubator-history. Infant,premature.

PALABRAS CLAVE: Enfermería neonatal. Incubadoras-historia. Prematuro.

RESUMO: O estudo tem como objetivos descrever os diferentes tipos de incubadoras existentes até a primeira metade do século XX, e relacionar os cuidados de enfermagem aos prematuros quanto à utilização das incubadoras. Trata-se de um estudo histórico. As fontes primárias são dois artigos existentes na Biblioteca Nacional e três artigos da Revista Brasileira de Enfermagem nos anos 40 e 50; e as secundárias são os livros e artigos de periódicos nacionais e internacionais relacionados à história da pediatria e da neonatologia. Os tipos de incubadoras são: fechadas, semifechadas e abertas. Quanto à assistência de enfermagem, constata-se dois momentos: os cuidados imediatos dispensados ao recémnascido na sala de parto e o seu transporte para o berçário, preferencialmente em uma incubadora, e os cuidados mediatos relacionados à assistência no berçário. Conclui-se que a incorporação das incubadoras influenciou diretamente os cuidados prestados aos prematuros, uma vez que exigiu uma maior especificidade na assistência.

\begin{abstract}
The objectives of this study are: to describe the different types of incubators that existed through the first half of the $20^{\text {th }}$ century and to list the kinds of nursing care given to premature babies concerning the use of incubators. It is a historical study. The primary sources are two articles that are found in the National Library, and three articles that were published in the Brazilian Nursing Review in the 1940s and in the 1950s; and the secondary sources are books and articles of national and international periodicals on themes related to pediatric and neonatology history. The types of incubators were closed, semi-closed and open. As far as the nursing care is concerned, one noticies two moments: the immediate care, relating to the first care given to the newborn in the delivery room and its transportation to the nursery, preferably in an incubator, and the intermediate cares, relating to the care given in the nursery. We conclude that it has influenced directly the care given to the premature babies since it requires a more specific assistance.
\end{abstract}

RESUMEN: El estudio de abordaje histórica que tuve como objetivos describir los diferentes tipos de incubadoras existentes hasta la primera mitad del siglo XX y relacionar los cuidados de enfermería a los prematuros en cuanto al uso de las incubadoras. Las fuentes primarias son dos artículos existentes en la Biblioteca Nacional y tres artículos de la Revista Brasileña de Enfermería de las décadas del 40 y del 50; y las fuentes secundarias son libros y artículos de periódicos nacionais e internacionais referentes a la Historia de la Pediatría y de la Neonatología. Los tipos de incubadoras son: cerrado, semi - cerradas y abiertas. En cuanto a la asistencia de enfermería, se verifica dos momentos: los cuidados inmediatos concedidos al recién nacido en la sala de parto y su transporte a la cuna de preferencia en una incubadora, y los cuidados imediatos relacionados a la asistencia en la cuna. Se concluye, que la incorporación de las incubadoras influenció directamente en los cuidados brindados a los prematuros, una vez que exigió una mayor especificidad en la asistencia brindada.

Endereço:

Isabel Cristina dos Santos Oliveira

Rua Domingos Mondim, 41 apt.106

21920-060 - Tauá, Ilha do Governador, Rio de Janeiro - RJ

E-mail: chabucris@ig.com.br
Artigo original: Pesquisa

Recebido em: 15 de fevereiro de 2004

Aprovação final: 24 de junho de 2004

Texto Contexto Enferm 2004 Jul-Set; 13(3):459-66. 


\section{INTRODUÇÃO}

O interesse e a preocupação pela condição da criança, por parte do poder público, juristas, educadores e médicos, manifestou-se a partir da segunda metade do século XIX até meados do século XX, em especial na transição de um para outro século. Nesse período houve um crescimento da valorização infantil no Brasil, despertando em determinados grupos a idéia de proteção, sendo que tal fenômeno predominou a partir do século XVII, na Europa.

Assim, a criança tornou-se o centro das atenções e cuidados e com isso impulsionou o desenvolvimento de uma assistência preventiva infantil voltada para os cuidados com a saúde física e, também, com a educação moral, baseados nos princípios de higiene pública ${ }^{1}$.

Vale destacar que "os serviços de saúde ampliaram-se, porém, não existia ainda um preparo adequado do pessoal para atender à população. E se o século XIX foi marcado por importantes realizações no campo da assistência médica à infância, pouco se fez na área da Enfermagem" "2:9.

No Brasil, constata-se que nas primeiras décadas do século XX houve a valorização da condição infantil que coincidiu com o aumento pelo interesse das necessidades dos prematuros e com a importação de incubadoras para cuidar dessas crianças.

A motivação em desenvolver este estudo justificase pelo interesse em entender a incorporação das incubadoras no cuidado de enfermagem aos prematuros.

Vale destacar que este estudo pode ser inserido na "tecnologias do cuidado", que na classificação das tecnologias específicas de enfermagem, "são todas as técnicas, procedimentos, conhecimentos utilizados pelo enfermeiro no cuidado" $3: 140$.

O estudo tem como objeto a assistência de enfermagem aos prematuros que necessitavam de incubadoras na primeira metade do século XX. Este recorte temporal se justifica pela incorporação das incubadoras no Brasil e pela intensificação das importações deste equipamento.

Os objetivos do estudo são descrever os diferentes tipos de incubadoras existentes até a primeira metade do século XX, relacionar os cuidados de enfermagem aos prematuros quanto à utilização das incubadoras e analisar a assistência de enfermagem aos prematuros que necessitavam de incubadoras.

Trata-se de um estudo com uma abordagem histórico-social. Os dados foram obtidos através de fontes primárias escritas e fontes secundárias. As fontes primárias escritas são dois artigos existentes no acervo da Fundação Biblioteca Nacional, e três artigos publicados na Revista Brasileira de Enfermagem das décadas de 40 e 50 . As fontes secundárias correspondem aos livros de história da pediatria no Brasil e exterior; livros e teses referentes à neonatologia e pediatria e artigos de periódicos nacionais e internacionais pertinentes a incubadoras e cuidados específicos aos prematuros, entre outras.

Para a análise das fontes primárias escritas processou-se a classificação temática por assunto.

\section{O ADVENTO DAS INCUBADORAS NO EXTERIOR E NO BRASIL: PERSPECTI- VAS PARA O CUIDADO DE ENFERMA- GEM AOS RECÉM-NASCIDOS (1839-1903)}

Este item destaca as primeiras incubadoras criadas para cuidar dos recém-nascidos prematuros no exterior e no Brasil, no período compreendido entre 1839 a 1903, ou seja, desde o primeiro protótipo até a importação deste equipamento para o Brasil.

Para se evitar a hipotermia, durante muito tempo, foram empregados diversos recursos para manter a temperatura corporal do prematuro, que consistiam na aplicação de envoltórios de algodão e no uso de botijas quentes. No Brasil, utilizava-se, ainda, as bolsas de água quente, e até mesmo o "tijolo quente", em áreas rurais. No entanto, estes meios improvisados não eram totalmente eficazes e podiam causar danos a integridade da pele dos recém-nascidos como queimaduras, uma vez que sua pele é muito delgada. Apesar disto, estes meios foram os primeiros passos para o desenvolvimento das incubadoras ${ }^{4}$.

Stéphane Etienne Tarnier, professor e obstetra parisiense, foi o responsável pelo desenvolvimento da primeira incubadora, ou seja, o inventor deste equipamento ${ }^{5-6}$. Por outro lado, acredita que "Budin e seu aluno Couney foram os pioneiros na construção da primeira incubadora destinada aos cuidados da criança prematura[...]"

Entretanto, a idéia de empregar um meio análogo ao usado na incubação artificial de ovos existe desde o século XVI, quando o pai do filósofo italiano Fortunius Liceti, que era médico, utilizou este recurso para salvar seu filho, que nasceu prematuro. Em 1839, o médico da Imperatriz da Rússia, Von Rühl, utilizou uma banheira para manter os recém-nascidos da Casa dos Expostos, de San Petersburgo, em um ambiente 
artificialmente aquecido ${ }^{4}$.

Partindo, também, desse pressuposto, em 1857, o professor Denucé, de Bordeaux, na França, produziu um berço incubador. A estrutura desse berço era constituída de zinco e formada por fundo e paredes duplas. Entre as paredes e o fundo, existia um espaço onde era armazenada água aquecida. Os bordos superiores das paredes eram integralmente unidos, sendo que num dos bordos havia um funil que provavelmente era destinado à introdução de água quente. $\mathrm{Na}$ porção inferior do berço, era acoplada uma torneira, que possivelmente escoava a água resfriada.

Em 1878, Tarnier convidou um funcionário do Zoológico de Paris para desenhar uma incubadora semelhante àquela usada no zoológico como chocadeira de ovos de galinha, e, em 1880, Tarnier apresentou sua incubadora, a qual instalou na Maternidade de Paris. Esta incubadora recebeu o nome de seu criador, e era constituída de uma caixa de madeira, composta por uma dependência superior, na qual o recém-nascido repousava, e outra inferior, onde eram depositadas as botijas com água aquecida ${ }^{4-5}$. Cabe ressaltar que o modelo da incubadora de Tarnier sofreu modificações realizadas pelo médico Auvard* . Essas mudanças consistiam na alteração da forma de armazenamento da água aquecida, pois, enquanto no modelo Tarnier a água encontrava-se em botijas, no modelo modificado por Auvard a água era contida num depósito 4 .

Em 1882, o obstetra francês Pierre Budin começou a se interessar pelos estudos da fisiologia dos recém-nascidos, estimulando o aprofundamento nesta área, dando, posteriormente, origem à subespecialidade Neonatologia ${ }^{7}$.

O médico Credé criou uma banheira semelhante, em 1884. Pode-se supor que havia semelhança da banheira de Credé com as incubadoras descritas anteriormente.

A excelência do invento deveria ser dada a Von Rühl (1839), da Rússia, e a Denucé (1857), da França. Todavia, Crede, tendo a pretensão de mostrar-se inventor da incubadora, acusou Tarnier de plagiador. Esse fato é evidenciado pelo relato: “O cotejo das datas mostra que à Von Rühl (1839) e Denucé (1857) cabe a prioridade do invento, o que não impediu que Credé tivesse a veleidade de se supor o inventor da incubadora, atirando sobre Tarnier a pecha de plagiário" "4:7.

No final do século XIX, o êxito dos resultados clínicos obtidos pelas incubadoras foi tão grande que esses equipamentos passaram a ser destaque comercial, rendendo lucros aos seus elaboradores. A incubadora de Martin Cooney, seguidor do professor Budin, foi apresentada na Exposição de Berlim, em $1896^{6}$. Além da Europa, Cooney exibiu sua incubadora em feiras e exposições nos Estados Unidos. Em 1901, visitou esse país para divulgar seu equipamento na Exposição Pan-Americana, em Búfalo-Nova Iorque ${ }^{5}$. Cabe destacar que Cooney obteve grande sucesso quando salvou, em quatro décadas, mais de cinco mil prematuros utilizando as incubadoras em Coney Island.

Não é possível detalhar com precisão a época da incorporação das incubadoras no Brasil. Todavia, supõe-se que tenha sido no início do século XX, já que os médicos Jaime Silvado e Antonieta Morpurgo, refletindo sobre os notáveis resultados obtidos pelo uso das incubadoras no cuidado aos prematuros e com a preocupação quanto à ausência desse equipamento nas maternidades do Rio de Janeiro, propuseram ao diretor do Dispensário Moncorvo a organização de um Serviço de Incubadoras neste estabelecimento que, em 1903, tinha duas incubadoras da marca Lion. A criação desse serviço deu-se com base num folheto referente às incubadoras Lion, que foi trazido por Antonieta Morpurgo quando visitou o respectivo serviço em Paris ${ }^{8}$.

A incubadora Lion foi fundamentada nos mesmos princípios das primeiras incubadoras, ou seja, manter o recém-nascido prematuro num meio aquecido e, assim, reproduzir um ambiente semelhante ao vivenciado por ele no organismo materno?

A importação das incubadoras para o Brasil, assim como ocorreu em outras inovações tecnológicas, despertou crítica na área médica. Tais críticas baseavam-se na desnecessidade da utilização da incubadora no cuidado de prematuros, pelo fato de o Brasil ser um país de clima tropical. Contudo, os responsáveis pela iniciativa de importar as incubadoras contestaram com argumentos fundamentados na oscilação de temperatura, no Brasil, em alguns meses do ano e na comparação da temperatura ambiente neste país, nas estações mais frias, com os países de clima temperado. Eles demostraram que, no Rio de Janeiro, a temperatura atingia $12^{\circ} \mathrm{C}-16^{\circ} \mathrm{C}$ na época de inverno, confirmando, então, as razões infundadas das manifestações contrárias, pois essa condição térmica do ambiente era suficiente para provocar o resfriamento corporal do recém-nascido e, consequentemente, a morte do mesmo?.

*Aluno do obstetra Stephane Etienne Tarnier

Texto Contexto Enferm 2004 Jul-Set; 13(3):459-66. 
Havia a necessidade de promover o esclarecimento da sociedade brasileira quanto à existência das incubadoras e das vantagens proporcionadas por elas. Para auxiliar nesta divulgação, foi feito um apelo aos médicos e às parteiras para que os mesmos encaminhassem os recém-nascidos prematuros ao Serviço de Incubadoras do Dispensário Moncorvo, não se influenciando pelas críticas feitas ao uso de tais equipamentos?.

\section{AS INCUBADORAS E SUAS ESPECIFICI- DADES}

Este item ressalta os diferentes tipos de incubadoras existentes no Brasil na primeira metade do século XX, descrevendo os principais modelos que foram utilizados nesse período.

Após a importação da primeira incubadora para o Brasil, foram surgindo, no país, diferentes tipos de incubadoras com fabricação internacional e nacional. Essas incubadoras foram classificadas em dois tipos: o primeiro era as incubadoras totalmente fechadas que incluíam àquelas com ar condicionado e temperatura, umidade e oxigenação controlados automaticamente, como a Isolette, e aquelas não tinham ar condicionado e nem controle automático da temperatura, umidade e circulação aérea, como a Armstrong, e o segundo eram as incubadoras parcialmente fechadas, como a Caixa de Nobel $^{10}$. As incubadoras também foram subdivididas em três categorias distintas, as incubadoras fechadas, as semifechadas e as abertas ${ }^{11}$.

As incubadoras fechadas caracterizavam-se por permitirem o total isolamento do recém-nascido e, ainda, por apresentarem temperatura e umidade constante, reguladas automaticamente. Os cuidados aos prematuros eram prestados por meio de orifícios que possibilitavam a entrada das mãos, sem a necessidade da abertura da incubadora. Desta categoria, destacavam-se as incubadoras Sanitas-Super, Sanitas-Mignon, Sanitas Mod. S.A. e Mod. S.B., todas de fabricação nacional, e a Isolette produzida pela indústria norteamericana ${ }^{11}$.

As incubadoras semifechadas não permitiam o total isolamento do recém-nascido, já que para manuseálo era necessária a abertura da tampa da incubadora. Incluem-se nesta categoria as incubadoras da marca Sanitas Mod. S., de fabricação nacional, e a incubadora Hess e a Armstrong, de fabricação internacional ${ }^{11}$.

Em relação às incubadoras abertas, havia a completa ou parcial abertura na porção superior da incubadora e o aquecimento era feito na parte inferior, o qual podia ser elétrico ou através de água quente. Nes- se tipo de incubadora, a oxigenoterapia era realizada pelo emprego de máscara ou tenda de oxigênio. A indicação para essa categoria de incubadora acontecia somente para prematuros com peso superior a 2.000g. Faziam parte deste tipo os berços aquecidos e a incubadora Hess, cuja função da tampa da parte superior da incubadora a tornava um berço aquecido ${ }^{11}$.

Dos diversos tipos de incubadoras, a Isolette e a Armstrong foram os modelos amplamente utilizados no ambiente hospitalar, sendo que a Isolette teve grande destaque por sua facilidade no manuseio e por sua maior precisão, tendo, assim, o reconhecimento internacional.

Em 1938, o médico Charles Chapple desenvolveu, no Children's Hospital Filadélfia, a incubadora moderna, conhecida como Isolette. Chapple realizou esquemas e ensaios clínicos em colaboração com a Air Shields Company e seus colegas do hospital, entretanto, ele não publicou nenhum dos resultados obtidos $^{12}$. A Isolette tornou-se o modelo padrão para os cinquentas anos seguintes ${ }^{13}$. Não obstante isso, o nome Isolette foi incorporado à linguagem das pessoas que atuavam nos berçários como sendo sinônimo de incubadora?.

$\mathrm{Na}$ ausência de incubadoras em berçários, existiam dois tipos de improvisação de incubadoras e um de berço aquecido. O primeiro tipo de incubadora consistia numa estrutura com um compartimento inferior por onde eram introduzidas bolsas de água quente para manter o aquecimento em seu interior. O segundo era uma estrutura circundada por vidros, por onde era possível visualizar o prematuro, que obtinha o aquecimento através do uso de lâmpadas elétricas e a ventilação por meio da abertura parcial da parte superior da incubadora. O berço aquecido poderia ser improvisado com base na utilização de caixotes, cestos ou malas com forros de tecidos de algodão que possuíssem compartimentos, como bolsas, para armazenar garrafas, bolsas de água quente e tijolo quente, e, assim, garantisse o aquecimento do prematuro ${ }^{10}$.

\section{CUIDADOS DE ENFERMAGEM AOS RE- CÉM-NASCIDOS NAS INCUBADORAS: DESAFIO DA EQUIPE DE ENFERMA- GEM (1949-1957)}

Este item enfoca os cuidados de enfermagem aos prematuros, ressaltando a incorporação das incubadoras. Para alcançar esta finalidade, considera-se o discurso oficial das enfermeiras, correlacionado-o ao 
discurso médico referente às incubadoras e à equipe de enfermagem.

Com base na análise das fontes primárias e secundárias, é possível constatar dois momentos do cuidado de enfermagem aos prematuros: os cuidados imediatos, referentes aos primeiros cuidados dispensados ao recém-nascido na sala de parto e o seu transporte para o berçário, e os cuidados mediatos, diretas ou indiretas no berçário.

No que tange ao cuidado imediato, três pontos fundamentais foram destacados: manutenção da temperatura, estabelecimento e manutenção da respiração e controle das infecções. Imediatamente após o parto, a equipe de enfermagem devia fazer a laqueadura do cordão umbilical e desobstrução das vias aéreas superiores por meio da aspiração de mucosidades, e providenciar o aquecimento do prematuro que podia ser feito através do uso de incubadoras, berço aquecido por lâmpadas elétricas, lençóis ou cobertores esterilizados e aquecidos. Em seguida, eram feitos outros cuidados como a limpeza do corpo com água na temperatura de $39^{\circ} \mathrm{C}$, a utilização de nitrato de prata a $1 \%$ nos olhos, a inspeção simplificada para detectar anomalias congênitas, entre outros ${ }^{10-1}$. Recomendavase que os primeiros cuidados de enfermagem, ou seja, a laqueadura do cordão e a aspiração das vias aéreas superiores, fossem efetuados, rapidamente, no interior de uma incubadora ${ }^{14}$.

Após a realização minuciosa dos referidos cuidados, era necessário que o prematuro fosse transportado para o berçário, preferencialmente em uma incubadora, ou na ausência deste equipamento, em um leito aquecido ou em cobertores, também aquecidos, tendo a precaução de oferecer oxigênio e umidade necessária ${ }^{10-1}$.

No berçário, os cuidados de enfermagem eram prestados conforme as necessidades e deficiências dos prematuros. Assim, para planejar os cuidados de enfermagem, era necessário considerar cinco aspectos fundamentais: a manutenção da temperatura e da atividade respiratória; prevenção de infecções; alimentação; observação e vigilância permanente da criança ${ }^{10-1}$.

Tendo em vista os referidos aspectos, o primeiro cuidado da equipe de enfermagem no berçário era proporcionar ambiente tranqüilo para o repouso do prematuro por várias horas no interior da incubadora com oxigênio, evitando a manipulação e mantendo rigorosa observação durante 24 a 48 horas para identificar cianose, presença de secreção e sinais de hipotermia e de hipertermia ${ }^{10-1}$.
Cabe destacar que os prematuros com peso inferior a $1.500 \mathrm{~g}$ deveriam permanecer em observação por um maior tempo, que se finalizava quando eles conseguiam tolerar um período de 8 a 10 horas sem o auxílio da oxigenoterapia e temperatura ambiente do berçário ${ }^{11}$.

Durante todo o período de permanência do prematuro na incubadora, a equipe de enfermagem deveria aferir a temperatura no interior da mesma e a temperatura do prematuro em intervalos regulares, inicialmente de $4 \mathrm{em} 4$ horas, nas primeiras 24 horas, e posteriormente de 6 em 6 horas ou de 8 em 8 horas ao dia, de acordo com o estado clínico do recémnascido ${ }^{11}$. Entretanto, recomendava-se a verificação da temperatura apenas duas vezes ao dia, e ressalta-se que em prematuros com baixo peso a temperatura poderia apresentar um nível inferior a $35,5^{\circ} \mathrm{C}$, que se elevava conforme o aumento de peso ${ }^{11}$. A temperatura do prematuro era verificada por via retal, com o termômetro lubrificado e, em seguida, anotada na papeleta ${ }^{15}$.

Quanto à temperatura do ambiente interno da incubadora, ela devia ser regulada de forma a manter a temperatura corporal do prematuro entre $36^{\circ}$ e $37^{\circ} \mathrm{C}$. Assim, ela podia ser ajustada entre 26 e $29^{\circ} \mathrm{C}^{10-5}$.

A temperatura na incubadora estava relacionada com a capacidade termorreguladora do prematuro, a qual era proporcional ao peso da criança. Desse modo, estabeleceu-se a relação do peso do prematuro com os graus centígrados na incubadora, a saber ${ }^{11}$ : peso do prematuro abaixo de $1.250 \mathrm{~g}-25$ a $26^{\circ} \mathrm{C}$; de 1.250 a $1.750 \mathrm{~g}-29$ a $30^{\circ} \mathrm{C}$; de 1.750 a $2.250 \mathrm{~g}-27$ a $28^{\circ} \mathrm{C}$; e de 2.250 a $2.500 \mathrm{~g}-25$ a $26^{\circ} \mathrm{C}$.

Além da temperatura interna da incubadora, devia-se fornecer uma quantidade adequada de umidade, que variava entre 55 e $65 \%$. Algumas incubadoras já eram programadas para ofertar o grau de umidade indicado para os prematuros ${ }^{10-1}$.

Em geral, os prematuros necessitavam de oxigenoterapia contínua ou intermitente para reduzir o esforço respiratório e, assim, auxiliar na conservação da energia, principalmente os prematuros com peso menor que $1.800 \mathrm{~g}$. Grande parte das incubadoras apresentavam o mecanismo de aquecimento combinado ao de administração de oxigênio, como, por exemplo, uma câmara de oxigênio. Quando inexistia a combinação dos dois mecanismos na incubadora, o suprimento de oxigênio deveria ser feito através de funil ou tubo de borracha ou ainda por tenda de oxigênio improvisada ${ }^{10}$. 
A concentração de oxigênio suficiente para o prematuro era de 40 a $50 \%$, podendo alcançar níveis de 70 a $80 \%$ em situações de intensa cianose. Em geral, nos manuais das incubadoras, havia uma tabela que orientava a quantidade de oxigênio em litros por minuto necessária para se conseguir determinadas concentrações no interior da incubadora. O período necessário para realizar o aporte de oxigênio dependia de cada prematuro. Para a maioria, um tempo de 24 a 48 horas eram suficientes, enquanto que um grupo menor necessitava de um período de 8 a 15 dias para melhorar o quadro respiratório ${ }^{11}$.

Outra preocupação da equipe de enfermagem consistia na utilização minuciosa das técnicas de lavagem das mãos, isolamento, assepsia e anti-sepsia, para prevenir as infecções e/ou infecções cruzadas em prematuros e, consequentemente, reduzir os altos índices de morbimortalidade das crianças. A incubadora foi um elemento importante para o isolamento do prematuro, pois, além de proteger a criança contra as infecções derivadas do ar, ainda limitava a quantidade de pessoas que the dispensavam cuidados, evitando a contaminação cruzada.

Existiam, também, outros cuidados de enfermagem aos prematuros relacionados com a pele, os olhos, as fossas nasais, a cavidade oral, o coto umbilical, os órgãos genitais, bem como o vestuário e o peso ${ }^{10}$.

Antes de efetuar os referidos cuidados, a equipe de enfermagem previa, provia e preparava os recursos materiais e do ambiente, além de fazer uma adequada lavagem das mãos, vestir o avental e colocar a máscara para proteção. No caso de incubadoras semi-fechadas, a enfermagem devia aumentar ao máximo a temperatura da incubadora antes de levantar a tampa e fechar o oxigênio depois de abrí-la ${ }^{15}$.

A massa corporal dos prematuros era mensurada diariamente ou em dias alternados conforme orientação médica, tendo o cuidado de sempre tarar a balança e cobrí-la com papel de seda esterilizado. Quando os recém-nascidos encontravam-se em incubadoras que não possuíam balança própria, pesavase-os envolvidos em cobertores que deviam ser previamente pesados para que se pudesse fazer a diferença do peso obtido com o prematuro coberto com a medida do peso do cobertor. Após tal procedimento, devia-se registrar o peso resultante deste cálculo ${ }^{10-5}$. No entanto, ressaltava-se que a mensuração do peso não deveria ser realizada diariamente de forma rotineira. Para ele, a pesagem de duas a três vezes na semana era suficiente $^{12}$.
Constata-se a preocupação com o manuseio constante do prematuro, com a redução do aporte ventilatório e, ainda, uma possível mudança brusca de temperatura que poderia ocorrer com o transporte do prematuro da incubadora até a balança.

O banho era feito entre 2 e 48 horas após o parto, utilizando óleo mineral embebido em algodão. Essa higiene será repetida com um intervalo de dois em dois dias até que o prematuro apresente um peso superior a 2.500g; alcançando-se essa meta, poder-seia banhar a criança diariamente ${ }^{11}$. A limpeza do prematuro deveria ser feita diariamente no interior da incubadora, conforme a técnica do berçário. Ao executar esta técnica, a equipe de enfermagem deveria movimentar o recém-nascido o menos possível e apenas o indispensável ${ }^{15}$.

Após a pesagem e o banho, fazia-se a troca da roupa do prematuro, o que era indicada conforme as condições da criança e da temperatura ambiente. Em geral, quando os prematuros permaneciam em incubadoras fechadas, especialmente na Isolette, recomendava-se o uso apenas de fralda. Para incubadoras semifechadas, como a Armstrong, era recomendado a fralda e uma camisa, podendo ser também somente a fralda. No caso de incubadoras abertas, devia-se colocar o capote de Hess, em clima frio, e cobertores, em clima quente ${ }^{14}$.

Ao finalizar as tarefas, a equipe de enfermagem retirava, lavava e esterilizava o material utilizado e fazia a limpeza da incubadora com o fim de prevenir as infecções nos berçários. A limpeza do equipamento processava-se na parte interna e externa da incubadora e dos berços aquecidos, através da lavagem diária com água, sabão e um pano ou papel para cada incubadora. Para estruturas metálicas da incubadora e dos berços recomendava-se a utilização de álcool ou amônia. É importante lembrar que, após a alta do prematuro, a incubadora era higienizada com água e sabão e, em seguida, colocava-se para arejar durante 6 horas antes de preparar uma nova admissão $\mathrm{O}^{10-5}$.

\section{CONSIDERAÇÕES FINAIS}

Com base nas fontes primárias escritas, evidenciou-se que, a partir do século XIX, se intensificou o interesse pela condição de vida das crianças e, em especial, pelas questões que envolviam o cuidado ao recém-nascido prematuro. A preocupação com os altos índices de morbimortalidade e com a necessidade fisiológica de preservar o aquecimento dos prematuros 
resultou na criação das primeiras incubadoras. $\mathrm{O}$ advento e a incorporação desse equipamento articulado com o avanço nos estudos da fisiologia dos recémnascidos, foram os elementos determinantes para a assistência a esse grupo infantil.

O princípio da incubadora de manter constante a temperatura corporal do prematuro em um ambiente artificialmente aquecido existia desde o século XVI, quando o pai de Liceti, para salvar seu filho, empregou um meio análogo ao usado na incubação artificial de ovos. A partir deste princípio, foram criadas as primeiras incubadoras. Em 1839, Von Rühl aplicou uma banheira de forma a aquecer os recém-nascidos. Denucé, em 1857, sintetizou um berço incubador, e, em 1880, Tarnier instalou sua incubadora na maternidade de Paris. O médico Credé criou, também, uma banheira semelhante às outra citadas, em 1884.

A utilização das incubadoras trouxe excelentes resultados clínicos para os prematuros, principalmente, para os com baixo peso e/ou menor idade gestacional. Tal fato impulsionou o ingresso desse equipamento no mercado comercial, que, por conseguinte, se tornou um investimento bastante lucrativo.

Acredita-se que a importação das incubadoras para o Brasil tenha ocorrido no início do século XX, quando os médicos Jaime Silvado e Antonieta Morpurgo propuseram ao diretor do Dispensário Moncorvo, a organização de um Serviço de Incubadoras na instituição e adquiriram duas incubadoras do modelo Lion.

A importação da primeira incubadora, no Brasil, favoreceu a entrada de outros tipos de incubadoras. Além das incubadoras provenientes do mercado internacional, constata-se que, paralelo à evolução industrial, houve o aparecimento de incubadoras de fabricação nacional. Os diferentes tipos de incubadoras eram classificados em fechados, semifechados e abertos, os quais se diferenciavam pela capacidade total, parcial ou ausente de isolamento da criança e de manutenção da temperatura, do fluxo de oxigênio e da umidade.

Durante o início e meados do século XX, constata-se que a incubadora Isolette, produzida, em 1938, por Chapple, foi amplamente utilizada no meio hospitalar, tendo grande destaque por ser de fácil manuseio e ter maior precisão na manutenção da temperatura. Com isso, a Isolette adquiriu o reconhecimento internacional, tornando-se modelo padrão para os próximos 50 anos, sendo seu nome incorporado à linguagem hospitalar como sinônimo de incubadora.
A despeito das controvérsias e questionamentos acerca da invenção e do uso das incubadoras, pode-se concluir que a sua incorporação no atendimento aos prematuros, no exterior e no Brasil, foi um marco importante para a história da pediatria, em destaque, da neonatologia, visto que possibilitou o controle da temperatura corporal dos prematuros, reduzindo, portanto, o número de óbitos de recém-nascidos prematuros e aumentando a sobrevida dessas crianças.

A incorporação das incubadoras no cotidiano da equipe de enfermagem influenciou diretamente os cuidados prestados aos prematuros, uma vez que exigia uma maior especificidade na assistência.

\section{REFERÊNCIAS}

1 Rizzini I. Assistência à infância no Brasil: uma análise de sua construção. Rio de Janeiro: Universidade Santa Úrsula; 1993.

2 Oliveira ICS. Da mãe substituta a enfermeira pediatria. Rio de Janeiro: EEAN; 1998.

3 Nietsche EA, Leopardi MT. O saber da enfermagem como tecnologia: a produção de enfermeiros brasileiros. Texto Contexto Enferm 2000 Jan-Abr; 9(1):129-52.

4 Silvado J. Incubadoras. Rev Méd São Paulo 1903 Abr; 6(7):610.

5 Harrison $\mathrm{H}$. The premature baby book: a parents' guide to cooping and caring in first years. New York: St. Martin; 1946.

6 Figueredo Júnior I. Alojamento conjunto: histórico. Pediatr atual 1993 Set-Out; 6(15):89-93.

7 Avery GB. Neonatologia: perspectivas na década de 1990. In: Avery GB. Neonatologia: fisiopatologia e tratamento do recém-nascido. $4^{\text {a }}$ ed. Rio de Janeiro: Medsi; 1999.

8 Novaes HMD. Processo de desenvolvimento tecnológico em saúde: demanda e uso da tecnologia - o consumo hospitalar de São Paulo.São Paulo: Depto. de Medicina Preventiva da Universidade de São Paulo; 1990. Relatório técnico final.

9 Silvado J. As incubadoras. Rev Soc Med Cir RJ 1905 Abr; $7(8): 6-17$.

10 Martins HAL. Prematuros: cuidados de enfermagem. Rev Bras Enferm 1957 Mar;10(1): 66-78.

11Orlandi OV. Assistência ao prematuro. In: Fortes H. Terapêutica infantil. $5^{\mathrm{a}}$ ed. Rio de Janeiro: Atheneu; 1954. p.20-38.

12 Downes JJ. Evolução histórica, estado atual e desenvolvimento prospectivo do tratamento intensivo pediátrico. Clín Ter Intensiva 1992; 1:1-25.

13 Rocha JM. Pediatria, puericultura e medicina infantil: manual 
para estudantes e pós-graduados. São Paulo: Prociemx; 1965.

14 Martins HAL. Prematuros e recém-nascidos com complicações: cuidados gerais de enfermagem. Rev Bras Enferm 1957 Set;10(2):89-90.
15. Universidade de São Paulo. Escola de Enfermagem. Técnica de enfermagem - cuidado do prematuro na incubadora. Anaes Enferm 1949 Abr;2(2):76-8. 